\title{
7. After the count and after the fact: at the Darwin Census Management Unit
}

\section{Frances Morphy}

Before embarking on a description of processes at the Census Management Unit (CMU), I will present some interim comments about the organisational structure of the Indigenous Enumeration Strategy (IES). I will use the debriefing of the Census Field Officers (CFOs) in Darwin as a frame for doing so, for two reasons:

- in the course of the debriefing, the CFOs raised many of the issues that I would like to raise myself

- as the people who were responsible for the practical implementation of the IES strategy, their perspectives on what worked and what did not are significant.

That being said, I am presenting my comments from the point of view of an outside observer. I am interested here in what the CFOs perceived as the problems, as an element of my own analysis. They sometimes saw similar problems, but offered different solutions. In some cases, the recommendations that we make in Chapter 9 coincide with solutions offered by some or all of the CFOs. In other cases, they are different.

\section{The CFO debriefing}

The debriefing session took place on 15 September, when many of the CFOs were still attempting to complete the counts in their regions. In some respects, then, they had not had a chance to gain any distance from the process and some, like the CFO I had been shadowing, were feeling tired and discouraged.

The topic of time - or lack of it - featured prominently in the discussion. It was felt that their own training had been too compressed, that the time allowed for pre-census publicity in the communities was insufficient and that the Community Housing and Infrastructure Needs Survey (CHINS) work had interfered with their census publicity efforts. The time allowed for training the Community Coordinators (CCs) and collector-interviewers (CIs) was insufficient, given people's levels of skill and knowledge, but they saw problems in keeping people engaged in longer training sessions. Finally, given the size of their regions and the logistical difficulties involved in keeping track of what was happening at more than one place at a time, they felt under constant time pressure. There was no time and little opportunity to carry out the validation checks that they had been asked to do in the field. Most had been unable to complete the count in the six 
to seven-week window, and they were concerned about the implications of this for the accuracy of the count, given people's mobility.

All of these time pressures are symptomatic of one thing: the current remote IES arrangements are unviable. One could level criticisms of the individual sub-strategies employed by some of the CFOs, such as making too much use of vehicles and not enough use of planes. This does not, however, alter my view that, fundamentally, the CFO's job as currently constituted is inherently impossible. These CFOs were hard-working and conscientious people. They were putting in tremendously long hours under often physically demanding conditions - sometimes to the point of exhaustion - and they all felt that they were not able to do the job as well as they would have liked, or as was required.

In part, it is an issue of the resources that the Australian Bureau of Statistics (ABS) is willing to put into the IES. The CMU staff and the CFOs were unanimous in their opinion that the whole exercise was under-resourced. The more fundamental question is the uses to which the available resources are put. More resources might indeed be necessary, but they also need to be deployed differently. This issue is discussed in detail in Chapter 9.

Many of the CFOs were critical of the outdated technology that they had to operate with. Although they had been given laptop computers for the CHINS exercise, they were not allowed to keep them for the census enumeration. They were forced to carry around huge amounts of paperwork and, unless they took their own laptops with them, they were unable to keep up-to-date electronic reports as they went along. It would have been useful to have electronic access to databases such as the recently completed CHINS and the Discrete Indigenous Communities Database (DICD), and they would have been able to update the latter electronically as they went. They were happy with the level of support that the CMU had attempted to provide, but had to rely on fairly unreliable satellite phones as their main means of communication. Having spent some time at the Data Processing Centre (DPC) in Melbourne, I find the contrast between the high-tech environment there and the low-tech environment visited on the CFOs startling.

The CFOs were generally critical of the ABS publicity for the census; they felt there was not nearly enough targeting of the IES areas with IES-specific information. The mainstream information that was disseminated was more confusing than helpful. They also felt it was largely a waste of time to target publicity at the general population in Indigenous communities. The effort should be targeted more to local organisations, and more time should be spent training the CIs in an understanding of the purpose of the census, so that they in turn could explain it properly to the interviewees. Several were highly critical of the materials they had been given to use in training. The DVD was 'not relevant to the bush' and the storyboard was a 'shame job'. 
I am sympathetic to the CFOs' criticisms. The census remains an alien project to most people in remote Indigenous communities, and publicity campaigns of short duration will not change that. It is no longer enough to simply tell people that the census is a good thing because it measures levels of need in housing and other basic services. This has been true for decades, and census results, in the eyes of many, have had no discernable effect on the government departments responsible for delivering these services.

The CFOs and the assistant CFOs all felt that the introduction of the Assistant role was a good thing. As one of the CFOs put it: 'The [region name], that's a lonely place down there. You need someone to offload with. There are lots of reasons to be working in pairs.'

Having two people also increased flexibility, with the CFO sometimes being able to leave the Assistant to supervise the count at one community, while moving on to start the training at another. They felt, however, that the respective roles and responsibilities of the CFO and Assistant CFO had not been mapped out clearly enough, and that this was a potential source of tension between them. They also thought that the Assistant CFOs had been brought on board too late in the exercise.

There was a discussion about the role of CCs, which it was felt was not differentiated sufficiently from that of the CIs. One suggestion was that CCs should be recruited and trained much earlier, and that they should be given responsibility for-and paid for-publicity activities and the recruitment of CIs. There was universal agreement that the training offered to CCs in 2006 was inadequate. In practice, because time was short, they were not given any more training than the CIs. One person suggested that the TAFE system could be used to deliver an accredited community-based training course on working for the census as a CC.

The CFOs were also very critical of the payment system for the CCs and CIs - it was complex, cumbersome and inefficient. Getting the pay details organised took up a substantial amount of time on the training day. They felt that people lost motivation because their pay was so delayed. Recruitment and retention of CCs and CIs in sufficient numbers had been an almost universal problem. The CFO for the Yolngu-speaking area brought up the example of a community of 1500 people in which he had been able to recruit only four people.

On the subject of their own training, the CFOs felt that their comments during the training had been vindicated. Not nearly enough time was spent on the content and implications of the questions on the form. They were particularly concerned about the 'persons temporarily absent' (PTA) question, feeling that they had been ill-prepared to assist the CCs and CIs in how to make decisions about moving people from the PTA table into the main body of the form. This comment can be linked to another, made by one of the CMU staff, that the longer 
the count went on the more complex this problem became. She felt that the count needed to be concentrated into three weeks at most, but acknowledged that this would require major changes to the organisation of the IES, and better resourcing. She suggested that one way of achieving the count in a shorter time might be to employ many more CFOs and give them responsibility for much smaller areas.

The CFOs also had some thoughts about the organisation of the CMU. There were three main issues. They thought that there should have been an IES-specific unit within the CMU - the managers to whom they were reporting had too many roles that involved them in the mainstream count, and this sometimes made it difficult to get the support that they needed in the field (at least one of the CMU managers concerned agreed strongly). They were critical of the lack of coordination and communication between themselves and the Area Supervisors of the mainstream count, and they questioned whether the State Indigenous Manager's role had been sufficiently well defined.

All in all, the outcome of this debriefing session was a fairly comprehensive critique of the IES in 2006, with a variety of solutions proposed to particular problems. In Chapter 9, we draw from this critique and from our own field observations to make our recommendations.

\section{The post-count checking process at the Census Management Unit}

In the second part of this chapter, I focus on the preliminary checking of the Interviewer Household Forms (IHFs) at the CMU, and on certain global problems with the data that emerged from the process. Checking was undertaken mainly at weekends by members of the CMU staff. From time to time, some of the CFOs were present, and the opportunity was taken to put outstanding queries to them about the regions in which they had worked. Several of the CFOs spent some time at the CMU helping to process the forms themselves.

There were two main tasks in the checking process. The first was undertaken in order to ensure that the forms were in maximally good order before being sent to the DPC in Melbourne. I learned later in Melbourne that not all CMUs had done this, and the DPC staff was very grateful to the Darwin CMU for its efforts. That Darwin focused on this exercise can be attributed to the fact that the person overseeing the IES had herself worked in the DPC in Sydney in 2001, and therefore had an understanding of the issues that would cause problems at the DPC if they were not addressed in Darwin. I draw attention to this as a classic example of the usefulness of 'local knowledge'. Because this person had an understanding of the context into which the forms were being sent, she was able to ensure that the forms were well prepared for that context. 
The necessity for the second task emerged as a result of this 'grooming' process. It quickly became apparent that there were very large numbers of PTA, and that the CFOs, CCs and CIs had not been consistent across the board in the way that these people had been treated. Many who should have been moved 'into the form' (see Chapter 2) because they were unlikely to be counted elsewhere had not been moved, and the sheer number of PTAs raised concerns about whether even those who were said to be somewhere where they should, theoretically, have been counted, had in fact been counted at that place. The second task, then, was to deal with the problem of PTAs.

I will devote most of this chapter to that second problem. When it became obvious, I decided that among other things, I would focus on the forms from the area where I had observed the count, reasoning that by doing so I would be able to undertake a detailed quantitative analysis of the scale and nature of the PTA problem in that particular area.

\section{Grooming the forms}

When the boxes of IHFs from a particular Collection District (CD) arrived at the CMU, they should have had the following accompanying documentation: ${ }^{1}$

- Indigenous Community Information form from the DICD

- Interviewer Dwelling Checklists (IDCs) for Indigenous dwellings

- separate IDCs for non-Indigenous and non-private dwellings

- Master Dwelling Checklist (MDC)

- checklist for CFO.

On the last of these, which the CFO was expected to complete before sending the forms to the $\mathrm{CMU}$, the $\mathrm{CFO}$ was required to crosscheck, among other things, that:

- the DICD form had been updated with available information

- an IHF had been completed for each unoccupied dwelling

- IHFs had been checked for Indigenous status non-response and followed up if necessary

- IHFs had been checked for duplications between the PTA list at Question 11 and the people listed as present at Question 12

- IHFs had been checked for usual address (Question 15)

- Question 10 ('Are there any persons who live here most of the time but are away?') had been marked appropriately 'no' or 'yes'

\footnotetext{
1 Note that the checklists were said to be applicable to a 'community', whereas in fact the IHFs were grouped into CDs. This is a potential source of error. For example, some small outstations in the area where I made my observations were included in the wrong $\mathrm{CD}$, and one was nearly missed altogether (see Chapter 4).
} 
- each IHF had its correct CD number, Census Record Number and form numbers (where more than one form had been used for a dwelling), and that these corresponded with the numbering on the MDC and IDCs

- every dwelling on the MDC had been included on an IDC

- non-Indigenous dwellings and non-private dwellings had been listed on separate IDCs

- $\quad$ every dwelling listed on an IDC had been included on the MDC

- counts had been checked against available estimates (with a note that at minimum these were the 2001 Census and the 2006 CHINS figures).

In practice, many of these CFO checklists were not filled in, so the CMU could not know whether or not the CFO had carried out the checks asked for, and very little attempt had been made to update the DICD forms. In many cases, the IDCs were also missing. ${ }^{2}$

The community checklist for the CMU had two sections. One- theoretically to be completed while the CFO was still in the field-asked the CMU checker to make sure that the CFO had recorded and reported the counts for people, dwellings and unoccupied dwellings. At the CMU, these counts were then checked against available estimates and the CFO notified of discrepancies for follow-up. In practice, this happened after the forms had been returned to the CMU and the CFO was either no longer in the field or was in a different CD, necessitating in some cases a return to particular CDs, or a follow-up by phone or fax.

The second part of the CMU checklist involved inspecting the forms to ascertain whether the CFO had carried out all the checks that they had been asked to do on the CFO checklist. In addition, the checkers at the CMU were asked specifically to do the following:

- check the CD number on each form

- check that the number of male and female - and total-persons listed at Question 12 for each dwelling corresponded with the number listed on the MDC

- check that Question 18 on Indigenous status had been answered (and, if it had not been, to answer it in the positive if, on the evidence available on the form, the person was incontrovertibly Indigenous)

- check Question 15 (usual address)

- check that the details for PTA (Question 11) had been filled in

2 In the case of the region in which I observed the count, this was because the contents of these lists had changed so often that they were almost useless as a record of what each CI had done. I suspect this could have been the case generally (see also Chapters 5 and 6). 
- check that people who had been moved to Question 12 ('inside the form') had also been crossed off the PTA table, and that the details of their sex and date of birth had also been transferred inside the form

- where a dwelling had people listed as PTA, but no one listed at Question 12, the checkers were instructed to amend the status of the dwelling to 'unoccupied' on the MDC

- double-check that the grand totals on the MDC were correct.

All of these checks - and most of the checks that the CFOs were asked to undertake - were concerned ultimately with ensuring the consistency of the 'head count' aspect of the census and ensuring that as far as possible basic information about residence and Indigenous status had been recorded. All things being equal, they were designed to ensure that the forms sent to the DPC were internally consistent with respect to the number, sex ratio, usual residence and Indigenous status of the population. In terms of the accuracy of the count, the checks were initially more general in nature: the CMU was concerned if the population count for a particular $\mathrm{CD}$ or the number of dwellings for which forms existed was very different from the recently acquired CHINS data and/or the count at the 2001 Census. There were several cases in which CFOs were sent out again to investigate the reasons for discrepancies.

There were several issues where the checkers were told not to try to 'fix' discrepancies, for example, anomalies in ages and/or in the data on relationships between the members of the household. They were told that they had insufficient information to make changes and that the DPC had rules and procedures that applied in such cases. Again, this was an example of the application of prior knowledge about the context into which the forms were being sent.

\section{The 'persons temporarily absent' problem}

Another problem quickly emerged as a result of the detailed scrutiny of the PTA question. One of the first CDs to be scrutinised consisted of small communities that were close to a regional centre. Many of the PTAs on these forms were said to be 'shopping' or 'visiting family' in the regional centre. There was no way of knowing, a priori, whether these people had gone only for the day or whether they had gone to stay in the regional centre for a while, and might have been counted there. In this case, the CFO who had been in charge of this CD happened to be in the CMU for the day. His local knowledge of the communities was tapped. His judgment was that people from these communities normally just went into town for the day, and that, moreover, the enumeration in the regional centre happened at a different time from the enumeration in these communities, so on both counts it was unlikely that these PTAs would have been 'caught' in town. Accordingly, they were all moved to Question 12 on their forms. In this 
case, it was not thought necessary to go through all the forms for the regional centre to try to find people counted there.

It is notable that the CFO had not himself made these judgments about moving PTAs before the forms were sent back to Darwin. Had he done so-and had he asked the CIs to go back and collect full details for the PTAs who were moved inside the form - many more people in the population of this CD would have had their full details recorded. Unfortunately, I did not think to ask him why he had not taken this step. There are several possible reasons: he had not understood the circumstances under which PTAs should be moved; he had not checked the forms closely (as per the CFO checklist) before sending them to Darwin; he simply ran out of time and had to make a judgment that it was better to get the forms in by the deadline he had been set than to spend more time chasing the details of PTAs. Given that the original CIs who had completed the forms might no longer be available, this could have been a lengthy process.

Similar problems kept coming up. It became clear that many CIs had not followed the instruction - or had not been instructed clearly enough - to move people from the PTA table in clear cases where they would not have been counted elsewhere, such as being away at 'sorry business'. It was also clear that most CFOs had not questioned the CIs' original allocations of people to the PTA category. In most cases, the reasons for moving people-or not moving them-were undocumented.

It also became clear that the prolonged nature of the count, combined with the levels and range of mobility, posed real problems for judgments about whether PTAs were likely to have been counted elsewhere. Theoretically, it should have been possible to check the advance schedules that stipulated when each community in each $\mathrm{CD}$ was to be counted, so that, for example, a person listed as a PTA from a community counted in July, who was said to be at a community that was counted towards the end of August, stood a good chance of having returned home before the latter count took place. It would be reasonable then to move such a person back into the form. These judgments depended on schedules being adhered to and on the CFO (at the very least) being aware of the schedules for their own region and also for surrounding regions and major population centres such as Darwin and Alice Springs. The CIs who were assigning people to the PTA category certainly did not have this information and so were not in a position to make such judgments (see my comments in Chapter 4 on my own dilemma over this very point), and there is very little evidence that the CFOs attempted to make such judgments in the field. In any case, most of the advance schedules underwent substantial alterations in the field, so it was almost impossible, in practice, for any CFO to be aware precisely when communities outside their sphere of responsibility had been enumerated. 


\section{Some preliminary thoughts on tracking absent persons}

In future censuses some of these problems could be ameliorated-but not eliminated - by better documentation, on several counts. Firstly, it should be documented whether people who are said to be 'visitors' at a dwelling have been asked how long they have been visiting for and whether or not they have been counted elsewhere. ${ }^{3}$ Secondly, more details should be asked about where a PTA is, how long they have been away and when they are likely to return, and this should be documented. In particular, if a PTA is said to be in a large town - for example, Darwin or Alice Springs - it becomes well nigh impossible to crosscheck whether they have been counted unless some specific location within the town is recorded. Thirdly, the 'short form' used to enumerate the 'homeless' should allow for a person's usual place of residence to be recorded. Many people who go temporarily to Darwin and Alice Springs camp out. It is not possible to crosscheck whether such PTAs have been counted if their usual place of residence is not recorded (see Chapter 5).

The situation could also be ameliorated by better use of technology. If the schedule were in electronic form, and if it were kept updated by the CFOs, and if every CFO had access in the field to this database, it would be a straightforward matter to make judgments - in the field - about the likelihood of PTAs being counted elsewhere. The CFO would simply have to look at the database to see what progress had been made in the CD to which the PTA was said to have gone. If that $\mathrm{CD}$ was being counted at more or less the same time, the chances would be high that the PTA would be counted as a visitor there. In the case where a large number of PTAs were said to be in a particular community, it would be possible to check - in the field - whether they were being picked up there.

Thinking further along these lines, it might also be profitable to institute a 'funerals and festivals' database, which would also be updated constantly by the CFOs on the basis of local intelligence, detailing where and when ceremonies and festivals were taking place, and which communities were affected, in terms of being the site of such an event or the source of many attendees. Such a database would make planning around these events much more manageable.

Schedules set in stone at the beginning of the census exercise will never be an effective tool in the context of the remote Indigenous census. There are just too many contingent factors at play. I am suggesting here the use of modern technology as a tool to constantly update the schedule as contingencies come into play, and to keep all CFOs - and possibly the CCs as well, because they will be the source of much of the intelligence - updated on the situation, not only

3 If people say they have been counted, they should of course not be counted again, but if they say they have not been counted it removes the necessity for checking to see if they have been counted at their usual place of residence. 
in their own areas of responsibility but in neighbouring areas where 'their' PTAs are likely to be found.

\section{Patterns of mobility and migration}

In a snapshot such as the census, it is difficult to disentangle short-term mobility from longer-term migration. The feeling expressed by some at the CMU was that the count was down in remote areas, and that this most likely reflected migration since 2001 from remote settlements and homelands into regional centres and towns. There are, however, at least three other possibilities:

- that the 2001 count was inflated by double-counting and the 2006 count is a more accurate reflection of the real population in remote areas

- that the 2006 count in remote areas was an under-count in comparison with the 2001 count

- that short-term circular mobility between homelands, hub settlements and regional centres has increased in the intervening period, so that at any particular time fewer people are at their place of usual residence than was the case in 2001 .

To these we can add the following possibility: that all these variables are at play to different degrees in different regions of the Northern Territory. Given this situation, it seems to me very unlikely that census data can be used to find definitive answers to questions about mobility or migration. There are, however, certainly indicative patterns that are worth noting for further investigation at a micro-demographic level.

In some areas, particularly in the arid and semi-arid zones, there were many small isolated homelands where all — or nearly all — the dwellings were empty. There are at least two possible reasons. One is migration into larger settlements and towns, which could well be a result of the closure of small Community Development Employment Projects (CDEP) programs and which is likely to increase if the Commonwealth government implements the policies it has foreshadowed for what it considers to be 'non-viable' small homelands, such as ceasing to provide funding for housing. If migration is involved, one would expect a corresponding swelling in the 'non-visitor' population of towns and regional centres - subject to the proviso that considerable numbers of people might nevertheless call themselves 'visitors' because they are not in their own 'country'. In some areas, however, it could simply reflect the seasonal occupation of homelands, with people moving regularly between their homelands and bigger hub settlements.

In particular instances (such as those described in Chapter 4), the emptiness of a settlement could reflect a very short-term movement, for example, to the site of a funeral ceremony or a festival. Such examples seem to occur commonly everywhere. 
Another definite pattern emerged in the areas around service centres such as Tennant Creek or Mataranka. Here it was very common to find large numbers of PTA who were temporarily away in the service centre, shopping or accessing other services. This pattern was repeated on a smaller scale in all areas where a set of satellite communities looked to a particular hub community for basic amenities such as shopping, banking, health services and so on. In such cases, short-term mobility is a more likely explanation than migration, and in nearly all such cases the PTAs should have been put inside the form.

A pattern that was particularly discernable in the relatively densely populated Top End was intense levels of movement between adjacent settlements in culturally defined regions - such as, for example, the Yolngu-speaking region of Arnhem Land. In such regions, many PTA were said to be visiting relatives in nearby communities. Such patterns were also discernable in less densely populated regions, but more intermittently and over much larger areas (see Chapter 5). These patterns too are indicative of short-term mobility rather than migration.

In the next few years, government policy settings could well result in increased semi-permanent migration flows from remote settlements into towns. It will be a complex matter to disentangle the evidence for this migration from the 'noise' of mobility more generally, and given the complexity of the patterns described above it would be inadvisable to use census data as any kind of baseline measure. There is an urgent need for regional micro-demographic studies of the nature and causes of mobility and migration in remote Australia.

\section{Documentation after the fact}

When the extent of the PTA problem became apparent, the CMU manager attempted to introduce some consistency into the decision-making about who should be moved into the forms, and instructed the checkers to document their decisions on the CMU checklists. She also attempted to get a fix on the scale of the problem, while acknowledging that the CMU did not have the systems to do a proper evaluation. Since the IHFs were not as yet in electronic form on a database, it was extremely difficult and time-consuming to crosscheck for the presence of individuals on more than one form. For example, some large communities had 10 or more boxes of forms, so that if PTA from another community were said to be there, all 10 boxes had to be checked. The DPC manager nevertheless instituted a check on PTAs where this was practicable. In some areas it appeared, from my own observations of this process, that a majority of PTAs had not in fact been counted at the places where they were said to be visiting.

As long as people were listed as PTA at their usual residence, these discrepancies would not have serious consequences for the final estimated resident population 
(ERP) - if PTAs were counted back into their communities for the purposes of the ERP. In such a case, however, the total Indigenous ERP of the Northern Territory would be significantly different from the total population counted in situ, since many of these PTAs did not appear inside any form unless they were moved back in at the CMU. Since PTAs are not counted back for the purposes of the ERP - and only those listed as visitors in other communities are counted back to their community - there will be a considerable under-count, because of the many PTAs unlikely to have been counted as visitors elsewhere. ${ }^{4}$ There are also significant consequences for the quality of the data on all questions except the basic demographic variables of age and sex. There are no other data available for those originally listed as PTA on a form, and if they were not counted elsewhere as visitors then those data were not collected elsewhere either.

In a few cases, the manager of the CMU asked the CFO to go back and collect details of PTA who had been moved back inside the form, or to attempt to do so by phone, however, it was not possible to undertake this exercise across the board.

\section{The Arnhem Land case-study area}

I took the opportunity at the CMU to scrutinise thoroughly all the forms from the homelands where I had undertaken my observation of the enumeration, and also those from nearby hub communities, in order to get some idea of the scale of the PTA problem in that area, and to assist the CMU in making judgments about which PTAs to put back into the forms. I also attempted to ascertain how many people had been missed altogether and how many had been double-counted, based on my personal knowledge of the local population. The former was a harder task than the latter: noticing an absence is a very different task from finding two instances of a presence.

In Chapter 4, I detailed particular instances of double-counting that I picked up in the course of this exercise, and I will not repeat those here. There were several other instances, particularly of the kind where a person was listed as PTA in one community or homeland, and as a resident at another. I found that one small homeland (population 30) had been overlooked (it was then subsequently visited). I also found one 'vacant' dwelling at one homeland, where everyone had been absent at a funeral at the time of the count. It had been intended for a follow-up visit that never happened (the CFO phoned the household from Darwin and the IHF was duly filled in).

For the study-area homelands as a whole - after eliminating all known instances of double-counting - I found that the IHFs listed a total of 598 residents at their

4 As noted in Chapter 1, this observation seems to be borne out by the published results of the post-enumeration survey (PES) exercise, conducted for the first time in 2006 in discrete Indigenous communities; see ABS (2007) and Taylor (2007b). 
own dwellings, 105 PTAs and 51 visitors. On the raw figures therefore, 105 out of 703 usual residents (15 per cent) of the usual population were PTAs, and 51 out of 649 ( 8 per cent) of the people present at the count were visitors. ${ }^{5}$ It must also be remembered that these counts were already something of a fiction (see Chapter 4) - for example, the inhabitants of A3 and A4 had been counted 'as if' they were at home, but the forms were filled in at community A, where they were attending a funeral.

I then went through the process of trying to crosscheck whether all PTAs had in fact been counted elsewhere. In some cases, PTAs were said to be at another of the homelands within the group, so crosschecking was relatively straightforward. In other cases, I had to trawl through all the boxes of IHFs from the hub communities in the region. In still other cases, people were said to be further afield and it was not possible to check most of these because the relevant boxes had already been sealed, awaiting transport to Melbourne.

As a result of this exercise, 70 of the 105 PTAs were put back into the forms, either because they were definitely not counted at the places where they were said to have been, or, in the cases where crosschecking was not possible, because they were unlikely to have been counted-for example, because they were at a funeral. That is, in the case of this set of communities, two-thirds of those who were originally listed as PTA-or 10 per cent of the usual resident population - would not have been counted anywhere unless this exercise had been undertaken. For this 10 per cent of the population, only very basic information is available: their sex, age, Indigenous status and usual place of residence.

\section{Conclusion}

Although I cannot extrapolate from my detailed findings about the PTA problem in the Arnhem Land homelands to the Northern Territory more generally, the overwhelming impression that I gained from being present at the CMU checking exercise when other areas were under scrutiny was that the problem would have been of the same order in many places. This is the key issue that emerges from this particular census exercise. It is a complex dilemma and the solution - which will never be perfect - is also complex, depending as it does on changes at many stages of the census exercise and at many sites within the ABS as an organisation. In the concluding chapter, we elaborate on these findings and make some recommendations for changes that will go some way to addressing them in the lead-up to the 2011 Census. In Appendix B, we discuss aspects of the IHF form design that probably contributed to the problem.

5 These are not the real figures; I have, however, preserved the correct percentages. 